IdeAs

Idées d'Amériques

15 | 2020

Eau et gestion de l'eau dans les Amériques

\title{
Chile despertó. Mobilisations sociales et politisation
} au Chili

Antoine Faure et Antoine Maillet

\section{OpenEdition}

Journals

Édition électronique

URL : http://journals.openedition.org/ideas/8364

DOI : $10.4000 /$ ideas.8364

ISSN : 1950-5701

Éditeur

Institut des Amériques

Référence électronique

Antoine Faure et Antoine Maillet, "Chile despertó. Mobilisations sociales et politisation au Chili », IdeAs [En ligne], 15 | 2020, mis en ligne le 01 mars 2020, consulté le 25 mars 2020. URL : http:// journals.openedition.org/ideas/8364; DOI : https://doi.org/10.4000/ideas.8364

Ce document a été généré automatiquement le 25 mars 2020

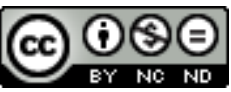

IdeAs - Idées d'Amériques est mis à disposition selon les termes de la licence Creative Commons Attribution - Pas d'Utilisation Commerciale - Pas de Modification 4.0 International. 


\title{
Chile despertó. Mobilisations sociales et politisation au Chili
}

\author{
Antoine Faure et Antoine Maillet
}

Le 18 octobre 2019, l'incendie d'une vingtaine de stations de métro lors de manifestations virant à l'émeute a marqué les esprits comme ne l'avait fait aucun événement politique depuis le retour à la démocratie au Chili. Au sein d'une "Amérique latine prise de convulsions », "l'oasis chilien » que décrivait le Président Piñera quelques jours auparavant (Cooperativa.cl, 2019) est lui aussi entré dans un processus de contestation de grande ampleur. Cet événement a ouvert une période d'intense mobilisation sociale et politique, dépassant les précédents établis lors des manifestations étudiantes de 2011, contre les retraites par fonds de pension en 2016 ou féministes en 2018. Dans chaque cas, on avait déjà parlé de leur caractère inédit dans le Chili post-dictature.

$2 \mathrm{Au}$ vu de ces cycles répétés de mobilisation, il est temps de cesser de caractériser le Chili comme un pays dépolitisé, récit dominant les travaux sur la transition démocratique et sur les enclaves autoritaires héritées de la dictature ${ }^{1}$. Il s'agit plutôt de s'interroger sur la nature de cette politisation, même si on commence seulement à prendre un peu de distance vis-à-vis de ce processus complexe ${ }^{2}$. A ces fins, nous revenons ici sur les dynamiques de (dé)politisation entre octobre et décembre 2019. Nous commençons par rendre compte des mobilisations sociales, véritable moteur de la séquence. Puis nous analysons la réaction de l'exécutif et les efforts des acteurs politiques pour s'arrimer au processus.

3 Dans ces différents domaines, nous présentons les multiples acteurs qui s'y meuvent et les jeux de politisation et dépolitisation à l'œuvre. A rebours de ce que la sociologie des crises affirme quant à une plus grande fluidité lors de mobilisations multi-sectorielles, ces trois domaines (social, gouvernemental et partisan) sembleraient maintenir ici une importante autonomie. On assisterait ainsi à différentes séquences où fluidité et autonomie se reconfigurent. Aujourd'hui absents ou du moins fortement bouchés, les canaux de dialogue sont tributaires, à nos yeux, de ces logiques de reconfiguration. Il s'agit là d'un enjeu important pour le futur politique du Chili. 


\section{Les mobilisations : la politisation de l'action collective}

Le mouvement débute avec les actes de désobéissance civile de nombreux lycéens de Santiago qui sautent les tourniquets de certaines stations de métro. Ils manifestent le rejet de l'augmentation des tarifs du transport public de 30 pesos décrété début octobre par le panel d'experts en charge du sujet (voir article de Larrouqué, dans ce dossier). Cette hausse pouvait pourtant paraître minime et ne s'appliquait pas au tarif étudiant.

Cette « fraude » (evasión) a vite pris de l'ampleur. La tentative policière de la contrôler a conduit à des affrontements, qui ont culminé le 18 octobre avec d'importants dégâts matériels et une paralysie du système de transports pour plusieurs jours. Cette ambiguïté entre action directe et délit s'est aussi manifestée, pendant plusieurs semaines, par le pillage (saqueo) de nombreux grands magasins (malls), supermarchés et chaînes de pharmacie entre autres, auxquels auraient participé des bandes organisées liées pour certaines au trafic de drogue.

6 Cette trame violente ne résume pourtant pas le phénomène. Les mobilisations massives se sont généralisées à l'ensemble du pays. On a ainsi vu des manifestations de très grande ampleur dans des parcours peu communs, en direction des quartiers aisés et du secteur financier de Santiago par exemple, mais aussi entre Viña del Mar et Valparaíso ou encore de La Serena à Coquimbo. Concepción est aussi apparue particulièrement combative, avec plusieurs cortèges par jour et des barricades quotidiennes au centreville. Dans un pays centralisé où les régions cultivent un certain antagonisme envers la capitale, cette propagation est surprenante.

7 Il est fort probable que les consignes initiales contre les abus du système aient eu un écho transversal, et que les déclarations pleines de mépris de différents ministres face à la désobéissance civile aient braqué le Chili dans son entier. Tout ceci a contribué à la rupture de "l'économie morale" de la domination (Thompson E., 1972) et s'est cristallisé, une semaine exactement après les incendies du métro, en des manifestations historiques, avec notamment plusieurs millions de manifestants défilant dans les rues à Santiago le 25 octobre. La critique généralisée des abus du système - par rapport aux retraites, aux salaires, à l'éducation, aux transports et à la santé - s'est aussi enrichie d'une demande spécifiquement politique pour une nouvelle Constitution (voir l'article d'Escudero et Gajardo dans ce dossier). La légitimité de la Constitution en vigueur, édictée durant la dictature de Pinochet (1980), et déjà modifiée 24 fois entre 1990 et 2010, était faible, mais le sujet apparaissait jusqu'alors comme relativement élitiste, à l'instar du processus constituant engagé durant le second gouvernement Bachelet (2014-2018). A partir de fin octobre, le projet d'une nouvelle Magna Carta devient populaire. On en discute notamment dans des cabildos (assemblées publiques), organisés entre voisins ou par des acteurs sociaux. Le thème s'enracine si fortement dans le débat public, qu'après avoir formulé un "agenda social ", le gouvernement se montre même enclin à ouvrir la discussion constitutionnelle (10 novembre). La majorité des parlementaires signent alors un accord pour une nouvelle constitution le 15 novembre. Unidad SocialMoneda Unidad Social

8 Entre les manifestations très souplement coordonnées et l'activité sociale et syndicale plus traditionnelle, de nombreux acteurs font aussi preuve de créativité et d'innovation dans les répertoires d'action. Les interventions visuelles (graffitis, murales, collages, etc.) transforment radicalement le paysage urbain des centre-villes. Certains emblèmes 
patrimoniaux sont objets d'intervention qui les resignifient. Ainsi à Temuco, capitale de l'Araucanie (terre d'origine des Mapuche), la statue de Pedro de Valdivia, "découvreur » du Chili, est décapitée, et sa tête remise entre les mains de la statue du martyr mapuche Caupolican. Par ce geste, la lutte n'est pas contre 30 pesos, ni contre 30 ans (comme le répétait un des slogans) mais contre 500 ans de colonisation. Par ailleurs, sous la pression des ultras et le relai de joueurs emblématiques, le championnat professionnel de football est conclu six journées avant la fin.

Les mobilisations ont même acquis un nouveau souffle avec les interventions du goupe Las Tesis (voir article de Ponce dans ce dossier). Leur chanson et chorégraphie «un violador en tu camino " (un violeur sur ton chemin) ont redonné un caractère massif et hautement contestataire aux manifestations. L'intervention est déclinée par de nombreux groupes sociaux (les Mapuche, les seniors, etc.) et obtient une importante visibilité internationale. On trouve ainsi en l'espace de deux mois un foisonnement d'initiatives, prises par une diversité d'individus, groupes et organisations plus ou moins formels, qui tous politisent les revendications de changements importants. Face à ce phénomène complexe et inattendu, comment l'exécutif a-t-il réagi et tenté de reprendre la main?

\section{Les rappels à l'ordre de l'exécutif}

Le pouvoir exécutif a montré des faiblesses dans l'analyse et la capacité de réponse audelà même de ce que pouvait imaginer ses plus féroces critiques. Le président Piñera a constamment privilégié une lecture à visée dépolitisante des mobilisations, uniquement présentées comme violentes et dont la responsabilité incomberait à des éléments non identifiés (encapuchados), exogènes et antisociaux. Son épouse, Cecilia Morel, a même été jusqu'à évoquer, sans humour, une «invasion étrangère, extraterrestre " dans un enregistrement WhatsApp. Une obscure manipulation médiatique a aussi tenté d'imputer les incendies à des agents cubains et vénézuéliens, allégations immédiatement démenties par le bureau du procureur. Sans parler de l'analyse des réseaux sociaux diffusée fin décembre et qui reprend cette thèse sur la base de tweets faits à l'étranger et attribuant en partie le désordre aux fans de... pop coréenne!

11 Rapidement, le président a décrété l'état d'urgence et déclaré «nous sommes en guerre ", avant que le Général en charge de la Défense nationale ne le démente en affirmant « je ne suis en guerre avec personne » (CNN, 2019). Piñera a ensuite été tenté par une stratégie conciliante, en annulant la hausse du prix des transports, puis en proposant le 23 octobre un «agenda social» très éloigné des demandes de la population. Sur son compte Twitter, il célèbre les manifestations du 25 en ces termes : elles « ouvrent de grands chemins de futur et d'espoir » (Piñera, 2019).

Cependant, terni par les initiatives législatives du gouvernement qui criminalisent les mobilisations sociales, le bilan en matière de droits humains est désastreux (voir article d'Aguilera dans ce dossier). On a compté au moins vingt-sept morts et quelque 3600 blessés, en très grande partie imputables aux violences policières. Outre des cas avérés de violences sexuelles suite à des interpellations, environ 360 personnes ont subi des blessures oculaires parfois irrémédiables. Ces exactions ont probablement contribué à renforcer les manifestations. La multiplication des missions internationales d'observation de ces violations des droits de l'Homme, et la force critique de leurs 
rapports (CIDH, HRW, HCDH, Allemagne, Canada, entre autres ${ }^{3}$ ), placent d'ailleurs le pays et son gouvernement dans une position peu commode sur le plan international (voir article de Parthenay dans ce dossier).

13 Pour reprendre le contrôle de l'agenda politique, le Président s'est défait de son ministre de l'Intérieur Andrés Chadwick, son cousin et cible des manifestants (voir article d'Alenda dans ce dossier), pour le remplacer par le jeune Gonzalo Blumel, qui a notamment annoncé lui-même l'ouverture du gouvernement à un processus constitutionnel. Marginalisé par l'accord conclu par les parlementaires et des taux de popularité à un chiffre, le président Piñera a maladroitement tenté de récupérer le processus constituant lors de la cérémonie de promulgation de la loi qui en fixe les modalités.

On a ainsi vu un pouvoir exécutif à la dérive, engoncé dans des idées de classe que la crise a fait ressortir. La stratégie privilégiée en décembre a, par exemple, visé à installer le contre-récit d'un "pouvoir narco » en pleine expansion. Cependant, les tentatives de dépolitiser la mobilisation à travers sa criminalisation n'ont pu stopper la dynamique sociale initiée le 18 octobre. Comment les acteurs politiques ont-ils essayé, de leur côté, de la traduire en actes?

\section{Des acteurs politiques chahutés}

Les acteurs parlementaires et les partis ont connu des difficultés similaires à l'exécutif pour gagner de l'emprise sur les mobilisations, mais y ont répondu de manière plus créative. Le personnel politique professionnel a initié un travail d'interprétation des phénomènes à l'œuvre, mais est resté inaudible pour la grande masse des manifestants. En conséquence, s'est creusée la distance entre partis politiques et acteurs sociaux déjà mise en évidence lors du mouvement étudiant de 2011 (Von Bülow M., et G. Bidegain, 2015).

Quelques rares acteurs ont toutefois su tirer leur épingle du jeu, notamment dans l'impulsion du processus constituant. Une fraction de la droite menée par Mario Desbordes et Manuel José Ossandón a ainsi pris ses distances avec Piñera et montré de l'empathie envers les demandes sociales. Les maires, regroupés en association, ont également voulu reprendre l'initiative en annonçant, dès le 7 novembre, la réalisation d'une consultation citoyenne en faveur d'une nouvelle Constitution. Cette proposition semi-formelle a certainement beaucoup pesé dans les dispositions constituantes de l'exécutif. Elle permet sans doute aussi d'expliquer la rapidité avec laquelle les parlementaires ont établi un accord signé par tous les partis représentés au Parlement, sauf le Parti Communiste et plusieurs factions du Frente Amplio (gauche). Indubitablement, les parlementaires ont fait preuve de capacité de réaction, mais n'ont pas totalement rompu avec les routines de la représentation politique, ce qui les a empêchés de traiter le problème de fond: le modèle de société et les inégalités. Les acteurs mobilisés critiquent d'ailleurs le fait que cet accord reprenne le système électoral actuel, lequel laisse peu de place aux candidatures indépendantes. Les partis de gauche et centre-gauche sont ensuite parvenus à des modifications marginales mais néanmoins symboliques, obtenant la parité de genre et des sièges réservés pour les communautés indigènes dans l'assemblée constituante. Un plébiscite aura lieu le 26 avril 2020 pour que les électeurs se prononcent sur la possibilité d'une nouvelle 
Constitution ainsi que sur la modalité du processus constituant (voir article d'Escudero et Gajardo dans ce dossier).

Il convient de rester prudent quant aux possibles traductions électorales des mobilisations durant le cycle politique qui s'ouvre maintenant, avec les élections locales d'octobre 2020, et les élections nationales de novembre 2021. Le Frente Amplio, qui émane des mobilisations étudiantes du début de la décennie, pouvait sembler le mieux placé pour établir une relation directe avec les manifestants. Toutefois, il semble aujourd'hui dans une phase de profond éclatement, ses leaders se retrouvant même conspués pour avoir signé l'accord constituant du 15 novembre, et plus encore pour avoir voté pour un projet de loi qui contenait diverses dispositions criminalisant les mobilisations.

On observe donc une déconnexion entre les mobilisations sociales et les acteurs politiques traditionnels, même dans ses franges les plus récentes, et ainsi un certain mystère quant à cette politisation loin et même contre les partis. Le débat qui s'ouvre en vue du plébiscite puis de la probable élection des constituants créera de fait de nouvelles instances de discussion où la relation société-personnel politique sera mise à l'épreuve. Il reste à espérer que la volonté de peser dans l'élaboration de la Constitution amène à des innovations politiques qui pourraient rendre compte de cette nouvelle politisation chilienne. L'héritage de la dictature et le récit de la transition postdictatoriale d'un côté, et les mouvements sociaux de la dernière décennie de l'autre, n'expliquent qu'en partie les mobilisations sociales et la politisation du conflit actuel. Il n'y a pas de fluidité totale entre les domaines, sans pour autant que chacun d'entre eux fonctionne de manière autonome. L'enjeu réside alors dans la reconfiguration du processus d'autonomisation du politique et des dynamiques de (dé)politisation dans un conflit social qui reste ouvert.

\section{BIBLIOGRAPHIE}

Arellano Francisco, Faure, Eyleen, López, María José, et al. « El “Nunca Más” que nunca fue : análisis de los cuatro informes sobre Derechos Humanos tras el 18/0 », CIPER, 20 décembre 2019, https://urlz.fr/bBqz, page consultée le 09/03/2020

CNN Chile, "Piñera: "Estamos en guerra contra un enemigo poderoso" ", Santiago, CNN, 21 octobre 2019, https://urlz.fr/bGKV, page consultée le 09/03/2020

Cooperativa.cl, « Presidente Piñera : Chile es un verdadero oasis en una América Latina convulsionada », Santiago, Cooperativa.cl, 9 octobre 2019, https://urlz.fr/bGKW, page consultée le $09 / 03 / 2020$

Piñera, Sebastian. « @sebastianpinera », Twitter, 25 octobre 2019, https://urlz.fr/bGKX, page consultée le 09/03/2020

PNUD, Los tiempos de la politización, Santiago, Programa de las Naciones Unidas para el Desarrollo, 2015. 
Thompson, Edward P., « The Moral Economy of the English Crowd in the Eighteenth Century », Past \& Present, $\mathrm{n}^{\circ}$ 5, 1971, p. 76-136.

Von Bülow, Marisa, et Germain Bidegain, « It Takes Two to Tango: Students, Political Parties and Protest in Chile (2005-2013) », in Paul Almeida et Allen Cordero Ulate (dir.), Handbook of Social Movements Across Latin America, New York, Springer, 2015, p. 179-194.

\section{NOTES}

1. Une exception importante est le rapport du PNUD (2015) intitulé Los tiempos de la politización. [https://urlz.fr/bGKO].

2. Le terme le plus utilisé pour décrire la période présente au Chili est estallido, l'explosion. S'il peut certes s'appliquer pour le jour même du 18 octobre, il nous semble incorrect pour la période entière, du fait de sa longueur et complexité.

3. Pour une comparaison de ces différents rapports, voir Arrelano F. et al. (2019).

\section{AUTEURS}

\section{ANTOINE FAURE}

Antoine Faure : École de journalisme - Université de Santiago du Chili antoine.faure@usach.cl

\section{ANTOINE MAILLET}

Antoine Millet est affilié à l'INAP - Université du Chili - COES

antoinemaillet@iap.uchile.cl 